\title{
Neutron emission spectroscopy of D plasmas at JET with a compact liquid scintillating neutron spectrometer
}

\section{Giacomelli, G.}

AIP Publishing

2018

Giacomelli , G , JET Contributors \& Ahlgren , T 2018 , Neutron emission spectroscopy of D plasmas at JET with a compact liquid scintillating neutron spectrometer . in Proceedings of the 22nd Topical Conference on High-Temperature Plasma Diagnostics . , 101113 , Review of Scientific Instruments, no. 10 , vol. 89 , AIP Publishing, Melville, NY, Biannual Topical Conference on High-Temperature Plasma Diagnostics, San Diego, California, United States , 16/04/2018 . https://doi.org/10.1063/1.5038549

http://hdl.handle.net/10138/326699

https://doi.org/10.1063/1.5038549

unspecified

submittedVersion

Downloaded from Helda, University of Helsinki institutional repository.

This is an electronic reprint of the original article.

This reprint may differ from the original in pagination and typographic detail.

Please cite the original version. 
WPJET1-CPR(17) 17645

R Zagorski et al.

\section{Modelling of JET DT experiments in ILW configurations}

Preprint of Paper to be submitted for publication in Proceeding of 16th International Workshop on Plasma Edge Theory in Fusion Devices (PET)

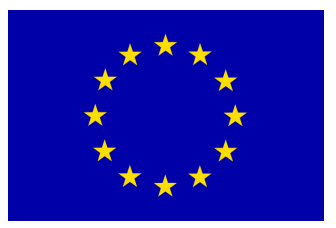

This work has been carried out within the framework of the EUROfusion Consortium and has received funding from the Euratom research and training programme 2014-2018 under grant agreement No 633053 . The views and opinions expressed herein do not necessarily reflect those of the European Commission. 
This document is intended for publication in the open literature. It is made available on the clear understanding that it may not be further circulated and extracts or references may not be published prior to publication of the original when applicable, or without the consent of the Publications Officer, EUROfusion Programme Management Unit, Culham Science Centre, Abingdon, Oxon, OX14 3DB, UK or e-mail Publications.Officer@euro-fusion.org

Enquiries about Copyright and reproduction should be addressed to the Publications Officer, EUROfusion Programme Management Unit, Culham Science Centre, Abingdon, Oxon, OX14 3DB, UK or e-mail Publications.Officer@euro-fusion.org

The contents of this preprint and all other EUROfusion Preprints, Reports and Conference Papers are available to view online free at http://www.euro-fusionscipub.org. This site has full search facilities and e-mail alert options. In the JET specific papers the diagrams contained within the PDFs on this site are hyperlinked 


\title{
Modelling of JET DT experiments in ILW configurations
}

\author{
R. Zagórski ${ }^{1, *}$, A. Czarnecka ${ }^{1}$, I. Ivanova-Stanik ${ }^{1}$, C. Challis ${ }^{2}$, and JET Contributors ${ }^{* *}$ \\ EUROfusion Consortium, JET, Culham Science Centre, Abingdon, OX14 3DB, UK \\ ${ }^{1}$ Institute of Plasma Physics and Laser Microfusion, Hery Str.23, 01-497 Warsaw, Poland \\ ${ }^{2}$ CCFE, Culham Science Centre, Abingdon, Oxon, OX14 3DB, UK
}

Received XXXX, revised XXXX, accepted XXXX

Published online XXXX

Key words JET seeding discharges, Integrated modelling, Core plasma, Edge plasma

\begin{abstract}
Numerical scan at constant $\beta$ shows that core and SOL radiations do not depend on the plasma current $\left(I_{p}\right)$. Whereas the SOL radiation increases with seeding, the core radiation however, does not continue to increase with seeding but rolls over at higher seeding rates in the simulations. The core plasma contamination by $\mathrm{W}$ ions is low $c_{W} \ll 10^{-4}$. When the seeding starts, an increase in radiation power leading to a reduction in $P_{\text {loss }}=\left(P_{a u x}-P_{\text {rad }}\right)$ is observed influencing the plasma confinement. The power scan at the constant $I_{p}$ indicates that core radiation, $P_{\text {plate }}, P_{S O L}$ (and even SOL radiation) saturates with seeding. In addition strong dilution with increasing seeding $\left(Z_{\text {eff }} \gg 3\right)$ and large $\mathrm{W}$ concentrations with increasing power are found. Comparing neon with nitrogen seeding, it was seen that neon leads to slightly larger total radiation than nitrogen. However, that is achieved with much higher plasma contamination $Z_{\text {eff }} \sim 4-5$ and dilution in case of $\mathrm{Ne}$, and simultaneously the power crossing the separatrix is lower for $\mathrm{Ne}$ than for $\mathrm{N}$, indicating better $\mathrm{H}$-mode performance in $\mathrm{N}_{2}$ seeded discharges.
\end{abstract}

Copyright line will be provided by the publisher

\section{Introduction}

A series of high power discharges $\left(P_{\text {aux }} \approx 21-26 \mathrm{MW}\right)$ using DT mixtures in ELM-free $\mathrm{H}$ mode was performed in 1997 during the JET DTE1 experimental campaign with carbon walls and divertor. A fusion power of 16.1 MW was achieved at 4.0 MA/3.6 T corresponding to the record fusion yield of $\mathrm{Q}=0.64$ [1]. It is planned to perform again the DT experiments at JET with the ITER -like wall (ILW) configuration (beryllium walls and tungsten divertor) during the DTE2 experimental campaign in 2018/2019 [2].The highest performance DTE1 plasmas were dynamic in terms of energy confinement, whereas plasma scenarios are being developed for DTE2 with the aim of maintaining high fusion performance in steady conditions with respect to the energy confinement and fast ion slowing down times. The energy confinement of JET plasmas has also been affected by the change in first wall materials [3] and the constraints for divertor compatibility with metal plasma facing components are more challenging. Consequently, the benefits of direct extrapolation from the highest performance DTE1 experiments to the conditions expected in DTE2 are limited. Therefore, the high performance plasma scenarios which are foreseen for DTE2 operation are based on either a conventional ELMy H-mode at high plasma current and magnetic field or on the so-called improved $\mathrm{H}$-mode or hybrid regime of operation with higher normalized energy confinement. In order to assess the plasma parameters in the DTE2 experiments COREDIV code [4] has been used to perform self-consistent core-edge simulations of JET DT plasmas. The code has been already successfully benchmarked with a number of JET discharges for both carbon and ILW configurations [5-8]. The preliminary COREDIV extrapolation of the reference ELMy H-mode shot \#87412 to high power $\left(P_{\text {aux }}=41\right.$ MW) DT operation shows good core plasma performance with fusion power of $\sim 6.5 \mathrm{MW}$ [9]. Although for hybrid plasmas, it might be possible to sustain 40MW of heating power for $\sim 5-6 \mathrm{~s}$ within the ILW divertor temperature limits [10], it would be advantageous to be able to use impurity seeding (e.g. Ne) to increase the margin with respect to the engineering limits. COREDIV preliminary simulations [9] showed that neon seeding leads to rather beneficial effect on the plasma performance allowing for relatively wide operational window in

* R. Zagórski E-mail: roman.zagorski@ifpilm.pl, Phone: +48 22638 1460, Fax: +48 226668373

** See the author list of X. Litaudon et al 2017 Nucl. Fusion 57102001 
terms of the amount of the allowed neon influx. That prediction however, should be confronted with the fact that impurity seeding dilutes fuel and, in most instances, reduces the fusion performance of JET plasmas in the plasma domain relevant for high performance in DTE2 [11].

In order to further investigate the possible range of operating scenarios COREDIV modeling is used to investigate effects of impurity seeding $(\mathrm{Ne}, \mathrm{N})$ in extrapolation to high power/current/field scenarios for the DT operation. In particular numerical scans have been performed in terms of different input powers $\left(P_{\text {aux }}=15-40 \mathrm{MW}\right)$, confinement factors $\left(H_{98}=0.8-1.2\right)$, plasma currents $\left(I_{p}=2.5-4.5 \mathrm{MA}\right)$, magnetic fields $\left(B_{T}=2.1-4.0\right.$ T) and plasma densities $\left(n_{e}=0.6-1.1 \times 10^{20} \mathrm{~m}^{-3}\right.$ ). As a first step (section 3), we have simulated plasma performance by increasing the input power and simultaneously some other parameters, keeping constant the $\beta_{N}$ value. Next, the power scan at constant current $\left(I_{p}=2.5 \mathrm{MA}\right)$ is presented in section 4 for two seeding gasses (neon and nitrogen). Final remarks and conclusion are given in section 5.

\section{Physical Model}

Simulations were performed by using COREDIV code which is based on an integrated approach coupling the radial transport in the core and the 2D multifluid description of the SOL. The interaction between seeded and intrinsic impurities as well as the effect of the impurities on the fusion power might significantly affect the particles and energy flows in the plasma and therefore the self-consistent approach is essential for a correct evaluation of the average power to the divertor plates. As this work forms a follow-up of our previous calculations, the detailed description and parameters used can be found in Refs. $[4,6,7,12]$ and only the main points of the model are reported here. In the core, the 1D radial transport equations for bulk ions, for each ionization state of impurity ions and for the electron and ion temperatures are solved. For auxiliary heating parabolic-like deposition profile is assumed and heating due to alpha power is calculated self-consistently taking into account the dilution effect due to helium and impurity transport. The energy losses are determined by Bremsstrahlung and synchrotron radiation together with ionization and line radiation losses. The energy and particle transport are defined by the local transport model with prescribed profile of transport coefficients taking into account the barrier formation in the edge region and which reproduces a prescribed energy confinement law [13]. In the SOL, the 2D fluid Braginskii like equations are solved in the simplified slab geometry but taking into account plasma recycling in the divertor region and sputtering processes due to all ions: $\mathrm{D},(\mathrm{T}, \mathrm{He}$ ), $\mathrm{Be}, \mathrm{Ne}$, and $\mathrm{N}$ (or $\mathrm{W})$ at the target plate. The model provides from a number of inputs like: the heating power, the average density and the confinement enhancement factor $\mathrm{H}_{98}$, all the plasma parameters of interest: the core temperature and density profiles, the effective ion charge $Z_{e f f}$, the radiated and fusion powers, the W flux and concentration and the plasma parameters in the divertor/SOL region. The code was run in a steady-state mode neglecting fast phenomena like e.g. ELMs. This corresponds to the experimental results averaged over energy confinement time.
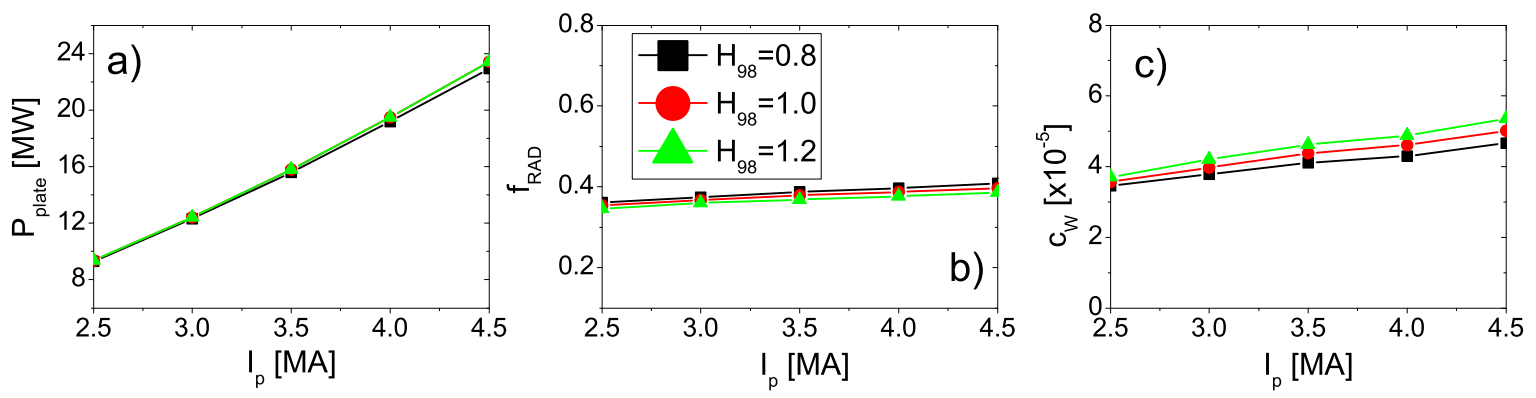

Fig. 1 Plasma parameters versus plasma current: a) power to the target plates $\left(P_{\text {plate }}\right)$, b) radiation fraction $\left(f_{\text {rad }}\right)$, c) W concentration $\left(c_{W}\right)$

\section{Simulations of the JET DT experiment - $\beta_{N}$ scan}

One of the main objectives of the coming deuterium and deuterium - tritium JET campaigns is to extend the performance of the ILW at higher plasma current ( $>2.5 \mathrm{MA}$ ) by fully exploiting the JET machine capability 
at high additional powers in the range of $40 \mathrm{MW}$. To reach these objectives, two main approaches are being pursued: (i) the ITER baseline scenario by simultaneously increasing the current, toroidal field and applied powers at $q_{95} \sim 3$ and $\beta_{N} \sim 1.82$, (ii) the ITER hybrid scenario at slightly reduced plasma current and higher $q_{95} \sim 3-4$ but at $\beta_{N}>2$ where confinement is increased at high beta through the interplay between the core and edge confinement optimisation [14].

In our study, first the expected level of radiation and heat loads at high current scenarios without impurity seeding is assessed, considering that the strike point sweeping might be sufficient to control the heat loads to the divertor targets. The following assumptions have been made: $q_{95}=3=$ const, $B_{T}=I_{p} \times 3.8 / 4.5\left(B_{T}\right.$ in $\mathrm{T}, I_{p}$ in MA), $n_{e} / n_{G}=$ const $=0.62, \beta_{N}=$ const $\left(=1.6\right.$ at $\left.H_{98}=0.8\right)\left(\sim W_{t h} / I_{p}^{2}\right), H_{98}=$ const, $P_{a u x} \sim I_{p}^{1.65}$, $n_{e}^{\text {sep }}=0.4 \times n_{e}$. Numerical scans have been performed for few values of plasma current (Table 1$)$ and $H_{98}$ $(=0.8,1.0,1.2)$ with corresponding $\beta_{N}$ values $\beta_{N}=1.6,2.0,2.4$, respectively.

Table 1 The main scan parameters

\begin{tabular}{llllll}
\hline$I_{p}[\mathrm{MA}]$ & 2.5 & 3 & 3.5 & 4 & 4.5 \\
$P_{\text {aux }}[\mathrm{MW}]$ & 15.2 & 20.5 & 26.4 & 32.9 & 40 \\
$B_{T}[\mathrm{~T}]$ & 2.11 & 2.53 & 2.96 & 3.38 & 3.80 \\
$n_{e}\left[10^{20} \mathrm{~m}^{-3}\right]$ & 0.62 & 0.74 & 0.86 & 0.99 & 1.11 \\
$n_{e}^{\text {sep }}\left[10^{20} \mathrm{~m}^{-3}\right]$ & 0.24 & 0.29 & 0.34 & 0.39 & 0.43 \\
\hline
\end{tabular}

It should be noted, that in order to keep $\beta_{N}$ constant, the power increases strongly with the current.

In the Fig.1, we show total heat load to the divertor $\left(P_{\text {plate }}\right)$, radiation fractions $\left(f_{\text {rad }}\right)$ and tungsten concentration $c_{W}$ versus plasma current for different $H_{98}$ values. It appears, that most of the global parameters (radiation levels, particle and energy fluxes) are very week function of $H_{98}$. However, the core and pedestal temperatures increase with $H_{98}$ as can be seen from Fig.2 where radial temperature profiles are shown together with the core radiation profiles, which are also affected by changes to $H_{98}$. With higher core electron temperature, the maximum of the radiation profile shifts from more central radial position $a / r=0.8$ to the pedestal region $a / r=0.93$ and might influence the pedestal parameters.

Without seeding, the radiation level is rather low ( $\sim 35-40 \%)$ (Fig.1b) and the corresponding heat load to divertor target plates might not be sustained in the steady state high current operation [10]. That indicates that strike point sweeping might not be sufficient to control the heat loads to target plates at peak plasma performance and additional impurity seeding might be necessary.

In order to check the effect of seeding on the plasma performance, simulations have been done assuming different levels of the Neon puff, while $\beta_{N}$ was kept constant. Only cases with $H_{98}=1$ and $I_{p} \geq 3$ MA are considered and the results are presented in Fig.3.
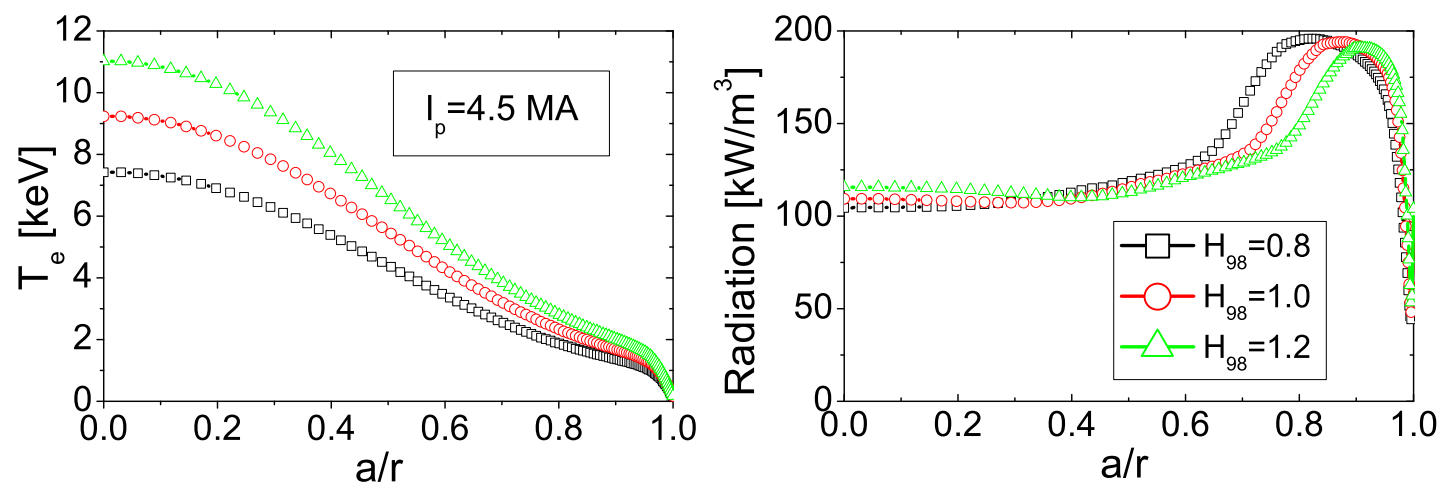

Fig. 2 Radial profiles of electron temperature and plasma radiation for the highest plasma current $\left(I_{p}=4.5 \mathrm{MA}\right)$

The influence of neon seeding on the plasma performance is quite positive. Already small amount of neon puff $\left(c_{N e} \geq 0.2 \%\right)$ leads to significant plasma radiation (Fig.3a) and reduction of the power to the divertor plates (Fig.3b) [11]. It should be noted, that the radiation fraction is independent on the plasma current and approaches $\sim 70 \%$ of the input power. That however is enough to reduce the heat load to the target plates to sufficiently 
low levels $(\sim 5 \mathrm{MW})$. The plasma radiation, is always dominated by the core tungsten radiation, which saturates already at relatively small neon concentrations $\left(c_{N e} \geq 0.2 \%\right)$ (note that our transport model does not consider strong $\mathrm{W}$ accumulation). We note also, that the tungsten concentrations are always low and stay below $\ll 10^{-4}$ level, in agreement with experimental findings. The SOL radiation increases almost linearly with seeding and at high seeding levels contributes significantly to the energy losses. Simultaneously, $Z_{\text {eff }}$ stays at reasonable level $(\lesssim 2.5)$ showing that the plasma dilution due to seeding should not be problem at the highest performance thanks to the plasma density increase with the power (in line with our constant $\beta_{N}$ assumption). The confinement factor,
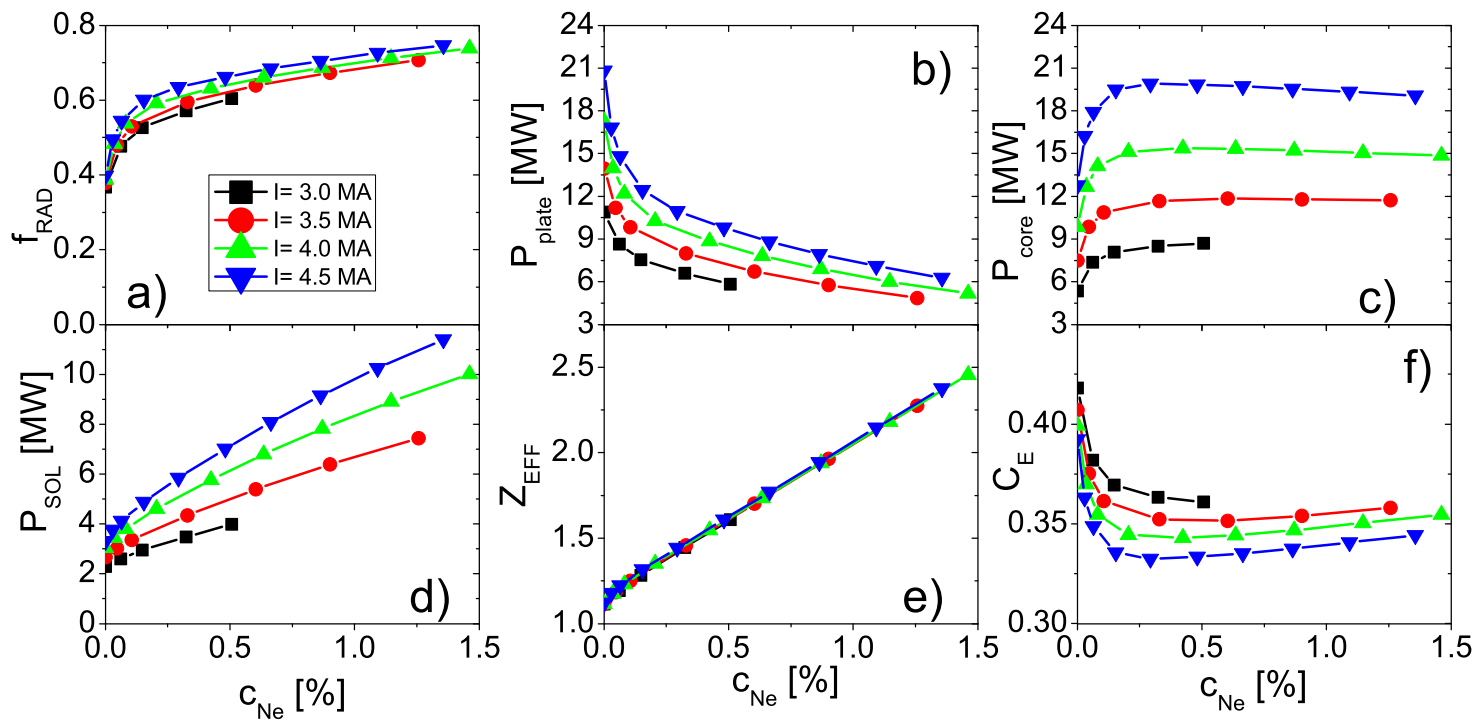

Fig. 3 Plasma parameters versus neon concentration for $\mathrm{H}_{98}=1:$ a) radiation fraction $\left(f_{\text {rad }}\right)$, b) power to the target plates, c) core radiation, d) SOL radiation, e) $Z_{\text {eff }}$, f) transport multiplication factor.

$H_{98}$ is kept constant in our simulations, which is achieved by feedback control of the transport coefficients. The corresponding control parameter $\left(C_{E}\right)$ is plotted in the Fig.3f. It can be seen that when the seeding starts the plasma transport (proportional to $C_{E}$ ) has to be reduced to keep constant $H_{98}$, which indicates that seeding might lead to some degradation of transport. Note, that the radiation from the plasma core increases while the H-factor is fixed, so the net energy confinement time $\left(\sim W_{t h} /\left(P_{i n}-P_{\text {rad }}\right)\right)$ increases and the local transport is reduced. However, at higher seeding ( $c_{N e}>0.25 \%$ ) confinement starts to improve with seeding, due to lower $\mathrm{W}$ radiation caused mostly by reduction of the $\mathrm{W}$ source (due to neon cooling of the divertor region).

\section{Power scan at $I_{p}=2.5 \mathrm{MA}$}

In the constant $\beta_{N}$ scan, the main discharge parameters were simultaneously changed, so the effect of specific parameter is somehow hidden in the results. In particular, the influence of the heating power might be of extreme importance and therefore simulation have been done changing only the input power keeping all the other parameters as for the $I_{p}=2.5 \mathrm{MA}$ case $\left(H_{98}=1.2\right)$ from the presented above $\beta_{N}$ scan.

Calculations have been performed for different levels of neon puff and three values of the auxiliary heating power: $P_{a u x}=15.2,27,40 \mathrm{MW}$ (with $\beta_{N}=2.4,2.9,3.3$, respectively) and are presented in the Fig.4. First, it can be seen that the radiation fraction (Fig.4a) saturates with seeding $(\sim 80 \%)$ and weakly depends on the heating power level. It is interesting to note, that at the constant heating power level, the core radiation $\left(P_{\text {core }}\right)$, power to the plate $\left(P_{\text {plate }}\right)$, power to SOL (not shown) and even the SOL radiation $\left(P_{S O L}\right)$ saturate with seeding. Seeding however leads to strong plasma dilution $\left(Z_{\text {eff }} \gg 3\right.$ ), at least for the considered relatively low density scenario $\left(n_{e}=6.2 \times 10^{19} \mathrm{~m}^{-3}\right)$. The degradation of the transport (Fig.4e) is larger for higher heating powers but it saturates with seeding, after initial strong change. Strong heating leads also to significant tungsten production and consequently high plasma contamination by $\mathrm{W}$ ions $\left(c_{W} \sim 3 \times 10^{-4}\right)$.

Up to now, only neon seeding was considered, since nitrogen seeding is not currently compatible with the JET tritium handling facility. However, in view of the successful JET experiments with $\mathrm{N}_{2}$ puffing, it is interesting 

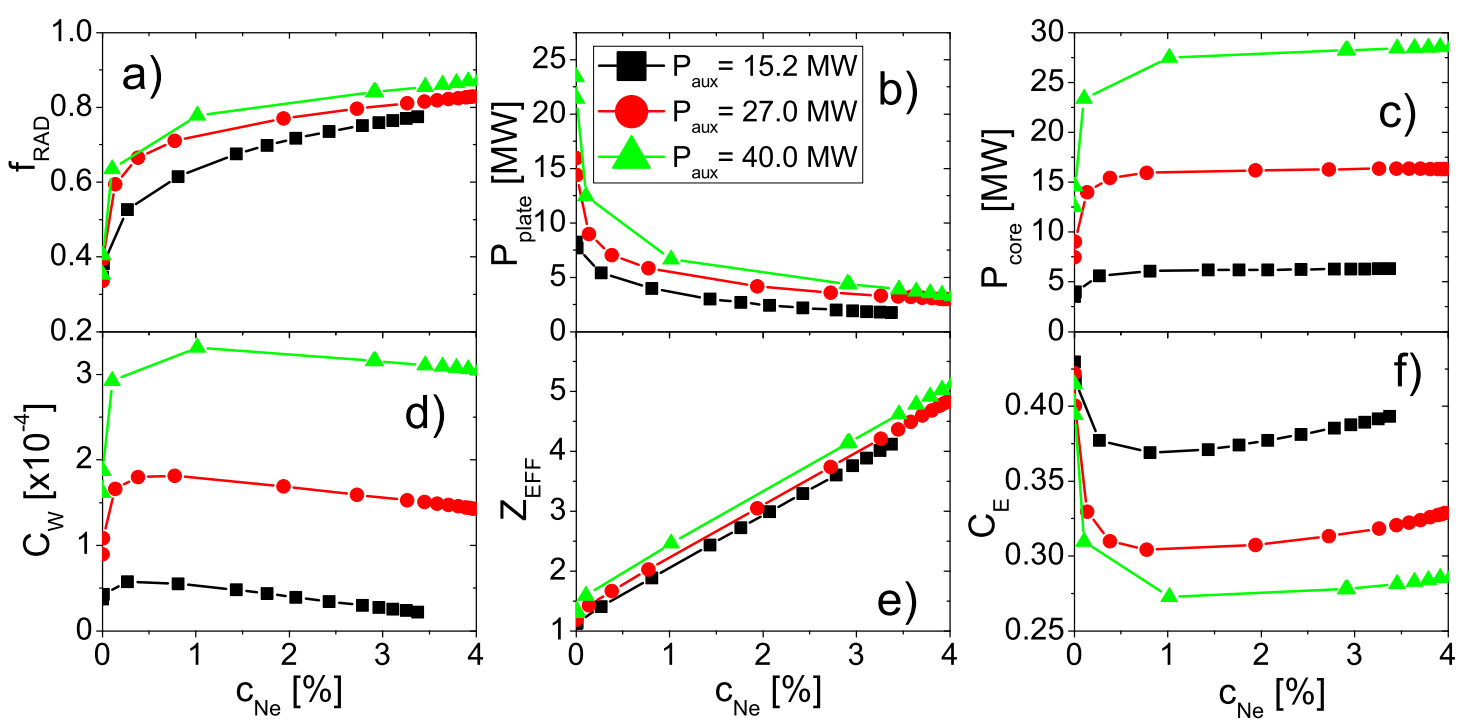

Fig. 4 Plasma parameters versus neon concentration for $\mathrm{H}_{98}=1.2:$ a) radiation fraction $\left(f_{\text {rad }}\right)$, b) power to the target plates, c) core radiation, d) tungsten concentration, e) $Z_{e f f}$, f) transport multiplication factor.

to compare both gases. That has been done for two heating levels $P_{a u x}=15.2,40 \mathrm{MW}$ and the results are shown in the Fig.5. It can be seen that neon leads to slightly higher total radiation than nitrogen, which leads to lower divertor heat fluxes in case of Ne. However, that is achieved with much higher plasma contamination $Z_{\text {eff }} \sim$ $4-5$ in case of Ne leading to strong plasma dilution, the fuel density $n_{i}(0)=4.8 \times 10^{19} \mathrm{~m}^{-3}$ compares with $n_{i}(0)=5.8 \times 10^{19} \mathrm{~m}^{-3}$ in case of nitrogen (at $P_{a u x}=40 \mathrm{MW}$ ). The power crossing the separatrix is lower for $\mathrm{Ne}$ than for $\mathrm{N}$, indicating better $\mathrm{H}$-mode performance in $\mathrm{N}_{2}$ seeded discharges. Also transport degradation with $\mathrm{N}_{2}$ seeding is weaker than for $\mathrm{Ne}$ (see Fig.5d), in particular for strongly heated plasmas. The simulations at fixed $H_{98}$ show that $\mathrm{N}_{2}$ seeding is favorable for energy confinement and core transport.
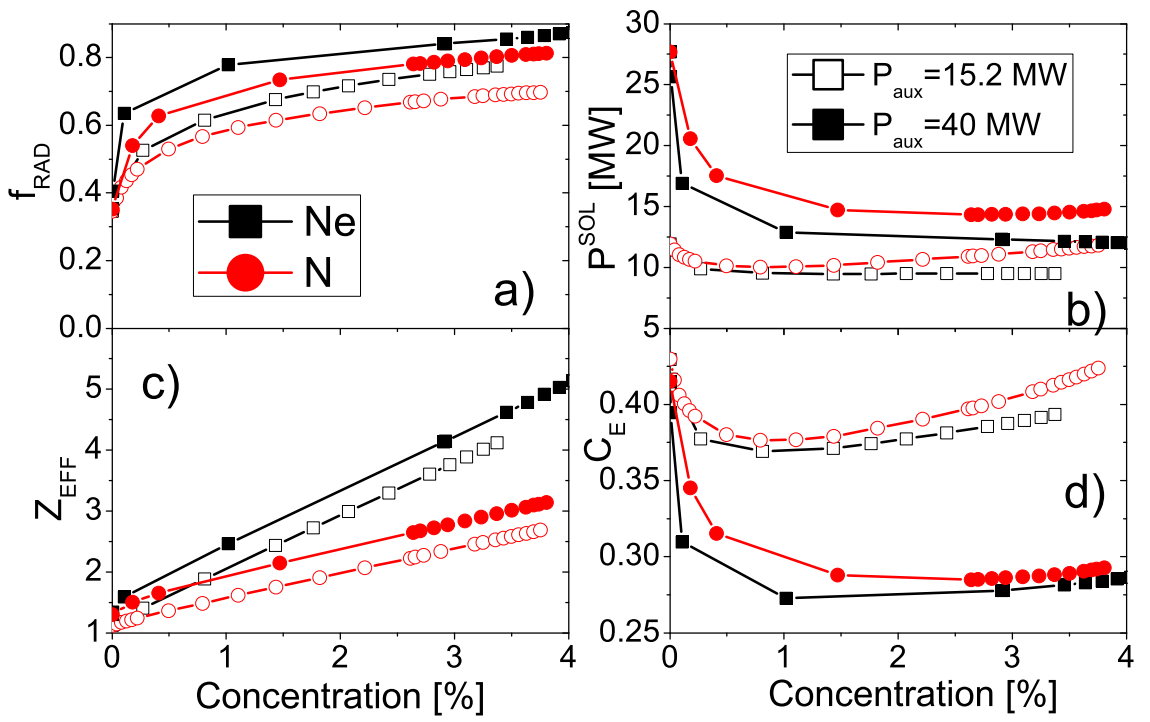

Fig. 5 Plasma parameters versus impurity concentrations (Ne-squares (black symbols), $\mathrm{N}_{2}$-circles (red symbols) for $P_{\text {aux }}=15.2 \mathrm{MW}$ (open symbols) and $P_{\text {aux }}=40 \mathrm{MW}$ (full symbols) : a) radiation fraction $\left(f_{\text {rad }}\right.$ ), b) power to SOL, c) $Z_{\text {eff }}$, d) transport multiplication factor. 


\section{Conclusions}

In order to investigate the possible range of operating scenarios COREDIV modeling was used to investigate effects of impurity seeding $(\mathrm{Ne}, \mathrm{N})$ in extrapolation to high power/current/field scenarios for the DT operation.

First, constant $\beta_{N}$ scan has been performed for so called hybrid scenario. The results of simulations show that without seeding, the radiation level is rather low $(\sim 35-40 \%)$ and the corresponding heat load to divertor target plates might not be sustained in the steady state high current operation. That indicates that strike point sweeping might not be sufficient to control the heat loads to target plates at peak plasma performance and additional impurity seeding might be necessary [10]. The effect of neon seeding on the plasma performance is quite positive and already small amount of neon puff $\left(c_{N e} \geq 0.2 \%\right)$ leads to significant plasma radiation and reduction of the power to the divertor plates [11].

We have analyzed also the influence of the heating power on impurity seeded discharges. It has been found that the radiation fraction saturates with seeding $(\sim 80 \%)$ and weakly depends on the heating power level. It is interesting to note, that at the constant heating power level, the core radiation, power to the plate, power to SOL and even the SOL radiation saturate with seeding. Comparing neon with nitrogen seeding, it was seen that neon leads to slightly higher total radiation than nitrogen, However, that is achieved with much higher plasma contamination $Z_{\text {eff }} \sim 4-5$ in case of $\mathrm{Ne}$, and simultaneously the power crossing the separatrix is lower for $\mathrm{Ne}$ than for $\mathrm{N}$, indicating better $\mathrm{H}$-mode performance in $\mathrm{N}_{2}$ seeded discharges. Also transport degradation with $\mathrm{N}_{2}$ seeding is weaker than for $\mathrm{Ne}$ in particular for strongly heated plasmas.

Acknowledgements The authors would like to thank Dr R.Neu and Dr T. P ̈̈tterich from IPP Garching for providing them with atomic data for tungsten.

This work has been carried out within the framework of the EUROfusion Consortium and has received funding from the Euratom research and training programme 2014-2018 under grant agreement No 633053. The views and opinions expressed herein do not necessarily reflect those of the European Commission.

This scientific work was financed within the Polish framework of the scientific financial resources in 2017 allocated for realization of the international co-financed project.

\section{References}

[1] M. Keilhacker, et al., Nucl. Fusion. 39, 209 (1999).

[2] L. Horton, et al., The JET DT Experiment, 12th International Symposium on Fusion Nuclear Technology, Jeju Island, South Korea, 14-18 September (2015).

[3] M.N.A. Beurskens, et al. PPCF, 55(12) (2013)

[4] R.Zagórski et al., Nucl. Fusion 53, 073030 (2013).

[5] J. Rapp, et al., J. Nuclear Materials, 337-339, 826 (2005).

[6] R.Zagórski, et al. J. Nuclear. Mater. 390-391,404 (2009).

[7] R.Zagórski, et al. Contrib.Plasma Phys.54(4-6), 442 (2014).

[8] R.Zagórski, et al. Contrib.Plasma Phys.52, 379(2012).

[9] R.Zagórski, et al. Contrib.Plasma Phys.56(6-8), 766 (201). / DOI 10.1002/ctpp.201610007

[10] S.Silburn, et al., PFMC 2017, submitted to Physica Scripta.

[11] C. Challis, et al., EPS 2017.

[12] G. Telesca, et al.,J. Nuclear Materials 438, S567 (2013).

[13] J. Mandrekas and W.M. Stacey, Nucl. Fusion 35, 843 (1995).

[14] X. Litaudon, et al., Nucl. Fusion 57 (2017) 102001 (41pp), https://doi.org/10.1088/1741-4326/aa5e28 